11

\title{
Фотодинамика переноса возбуждения носителями заряда в гибридной наносистеме InP/InAsP/InP
}

\author{
() А.С. Рубан, В.В. Данилов \\ Петербургский государственный университет путей сообщения Императора Александра I, \\ 190031 Санкт-Петербург, Россия \\ e-mail: rubananna@mail.ru
}

Поступила в редакцию 01.04.2021 г.

В окончательной редакции 01.04.2021 г.

Принята к публикации 09.04.2021 г.

\begin{abstract}
Приведены результаты обработки кинетики затухания люминесценции гибридной полупроводниковой наноструктуры InP/InAsP/InP с нанесенными коллоидными слоями квантовых точек (QD) CdSe/ZnS при возбуждении на длинах волн 532 и $633 \mathrm{~nm}$ и температурах 80 и $300 \mathrm{~K}$. Такая наноструктура характеризуется значительным увеличением длительности и интенсивности люминесценции нановставки InAsP. Механизм увеличения длительности люминесценции предположительно связан с взаимодействием коллоида QD $\mathrm{CdSe} / \mathrm{ZnS}$-TOPO с поверхностью InP, что ведет к образованию в запрещенной зоне новых гибридных состояний, энергетически близких к излучающему состоянию и способных захватывать электроны, что в свою очередь компенсируется возрастающей ролью процесса обратного переноса электрона, приводящего к росту длительности излучательной рекомбинации.
\end{abstract}

Ключевые слова: гибридные полупроводниковые наноструктуры, кинетика люминесценции, обратный перенос энергии.

DOI: $10.21883 /$ OS.2021.07.51087.2101-21

\section{Введение}

В последнее время интерес исследователей смещается в область полупроводниковых нанопроволочных конструкций [1-9]. Особый интерес представляет создание гетероструктур типа нановставка (QI) (или квантовая точка, QD) в теле полупроводникового нитевидного нанокристалла (НHK). QD, вставленные в полупроводниковые ННК с большей шириной запрещенной зоны, могут сушественно расширить диапазон их прикладных возможностей, например в квантовой обработке информации $[4,8]$. В работе [9] впервые приведены результаты спектральных и кинетических исследований ИК люминесценции новой композитной структуры, состоящей из HНK InP c QI InAsP (выращенных на подложке Si (111)) и нанесенных коллоидных QD CdSe/ZnS. Было выяснено, что нанесение на гибридную ННК InP/InAsP/InP квазиленгмюровского слоя триоктилфосфиноксида (ТОРО), содержащего коллоидные QD CdSe/ZnS, приводит к значительному увеличению длительности и интенсивности люминесценции QI InAsP.

Известно, что поверхностные лиганды играют существенную роль в динамике рекомбинации носителей заряда не только коллоидных QD, но и более сложных наноструктур [10,11]. Пассивация уменьшает количество поверхностных оборванных связей и, следовательно, сводит к минимуму вредный эффект низкоэнергетических ловушек для электронов или дырок. Ловушки увеличивают количество безызлучательных каналов релаксации, что в конечном итоге снижает эффективность флуоресценции QD. Полное определение и полный контроль излучательных характеристик QD - одна из ключевых задач исследователей наноструктур. Очевидно, что использование нанокомпозитов требует осознания характера межфазной фотодинамики и особенностей взаимодействий, которые связывают экситонные и поверхностные состояния, а также общих принципов, регулирующих люминесценцию в наносистеме.

Результаты исследований последних лет, приведенные в работах [6-16], показали, что фотолюминесценция (ФЛ) с разрешением по времени является эффективным количественным инструментом анализа фотодинамики безызлучательных процессов в полупроводниковых нанокристаллах, что значительно расширяет методические возможности исследователей. Особенностью кинетики затухания. люминесценции полупроводниковых наноструктур является их полиэкспоненциальность, отражающая сложную динамику возбужденного состояния [5,12-15]. С другой стороны, в силу отсутствия устойчивого формализма в обработке таких экспериментальных результатов полученные данные редко прямо соотносятся с конкретными фотопроцессами, происходящими в наносистеме [16-18]. В качестве альтернативы хорошо известным примерам простой подгонки кинетических кривых линейными комбинациями из двух или трех экспоненциальных функций в работах [5,19-23] было показано, что затухание ФЛ QD может быть описано как чисто радиационный процесс, конкурирующий с каналом передачи электронной энергии в колебательные состояния. При этом используют растянутую экспоненту (stretched exponential function) [24], и кинетика люминес- 
ценции описывается зависимостью $[19,20,22]$

$$
I(t)=I_{0} \exp \left[-\frac{t}{\tau}-A\left(\frac{t}{\tau}\right)^{\beta}\right],
$$

где $I_{0}-$ интенсивность люминесценции в начальный момент времени, $\tau$ - время жизни возбужденного состояния люминесцирующих молекул, $A-$ константа, зависящая от концентрации тушащих молекул, размерности среды и механизма тушения люминесценции. Такой подход справедлив и для коллоидных растворов QD, поскольку излучательная способность QD точек чрезвычайно чувствительна к окружению. При этом пассивация поверхности QD лигандами не исключает энергообмен со средой и существование явлений переноса. Работоспособность такого подхода была подтверждена в ряде публикаций $[19,21,22]$, в том числе при изучении фотодинамики люминесценции $\mathrm{CdSe} / \mathrm{ZnS}$, пассивированной слоем ТОРО. Еще более эффективным оказалось применение функции (1) к спектрокинетическим зависимостям более сложной наноструктуры InP/InAsP/InP с нанесенными на нее коллоидных QD CdSe/ZnS [5].

Традиционно неэкспоненциальный характер затухания люминесценции нанокристаллов аппроксимируется суммой нескольких экспонент с временами затухания $\tau_{i}$ :

$$
I_{\text {norm }}=\frac{\sum_{i}^{n} A_{i} e^{-t / \tau_{i}}}{n}
$$

где $I_{\text {norm }}$ - нормированная на единицу в начальный момент времени интенсивность затухания люминесценции; условие, накладываемое на амплитуды, $\Sigma_{i}^{n} A_{i}=1$. В то же время данный подход предполагает существование либо нескольких безызлучательно связанных метастабильных состояний, либо нескольких групп нанокристаллов с различной морфологией и разными временами затухания люминесценции $\tau_{i}$. Относительная доля этих состояний (или групп) в суммарной кинетике затухания как раз характеризуется коэффициентами $A_{i}$ при экспонентах. Особо отметим, что в ряде случаев экспериментальные кинетики затухания люминесценции хорошо аппроксимируются как суммой двух, так и трех экспонент (2). Очевидно, что использование нанокомпозитов требует понимания характера межфазной фотодинамики и прежде всего особенностей взаимодействий, связывающих экситонные и поверхностные состояния. Результаты работ $[5,19,23]$, где выполненные спектрокинетические результаты были аппроксимированы растянутой экспоненциальной функцией (stretched exponential function) привели к заключению, что при двух- и трехэкспоненциальном разложении (2) наименьшее значение $\tau_{i}$ чаще всего соответствует длительности естественного распада возбужденного состояния, скорректированного на процессы тушения. Вторая по значению величина $\tau_{i}$ соответствует подпитке возбужденного состояния за счет процессов внутренней конверсии, синглет-синглетного переноса (как излучательного, так и ферстеровского типа (FRET)) и рассматриваемого в этой работе так называемого „обратного переноса“ возбуждения, захваченного ловушкой, на излучающее состояние. Третью компоненту разложения можно связать с переносом возбуждения на излучающий уровень с более глубоких ловушек [21], темных состояний или при усложнении механизмов ретрансляции возбуждения.

Предложенный подход позволяет проанализировать результаты, полученные ранее, с учетом работ [10,23], где приводится наблюдение эволюции во времени спектра ФЛ QD при импульсном возбуждении, регистрируемой в наносекундной и микросекундной временных областях. В течение первых 20-50 ns после возбуждения наблюдается красное смещение спектра, что и вызвано диффузией экситонов по механизму FRET, после чего эта эволюция спектра останавливается и даже превращается в синий сдвиг. По мнению авторов [10,23], это демонстрирует эффект обратимого хранения носителей заряда, который временно замораживает QD в состоянии, из которого FRET не может произойти. То есть носители заряда имеют конечную вероятность оказаться на ловушках прежде, чем может произойти спонтанное излучение и/или FRET. Как только носитель заряда захвачен, передача экситона с помощью FRET временно блокируется. Высвобождение захваченных носителей заряда восстанавливает экситонное состояние. Расчеты авторов говорят о существовании сильных поверхностно-лигандных взаимодействий, приводящих к существенному перераспределению заряда и влиянию поляризации на поверхность. Эти эффекты приводят к развитию гибридизированных состояний, для которых электронная плотность распространяется на кластер и лиганды. В то же время в работе [9] показано, что анализ процесса замораживания носителей в гибридной системе может быть проведен с помощью модели, основанной на использовании разложения (2) и растянутой экспоненты (1) и описывающий так называемый „обратный перенос“ фотогенерированных носителей заряда. В этой модели при трансляции возбуждения любой захват носителей на ловушку рассматривается как тушение возбужденного состояния излучающего центра, а деактивация ловушки с последующей излучательной рекомбинацией - как обратный перенос. Такой подход, в частности, показал [9], что кинетика затухания люминесценции самой QI InAsP лучше всего описывается в рамках так называемой „модели контактного тушения“ [20].

В настоящей работе, основываясь на спектрокинетическом подходе, продолжено изучение влияние длины волны возбуждения на фотодинамику гибридной полупроводниковой наноструктуры InP/InAsP/InP [9,25].

\section{Экспериментальные условия}

Объектами изучения являлись как ННК, синтезированные методом молекулярно-пучковой эпитаксии, так и их композиции с осажденными на поверхность QD $\mathrm{CdSe} / \mathrm{ZnS}$, стабилизированными ТОРО (575 nm - мак- 
Таблица 1. Возбуждение на длине волны $532 \mathrm{~nm}$. Регистрация на длине волны излучения $\mathrm{InP}(900 \mathrm{~nm})$

\begin{tabular}{c|c|c|c|c}
\hline \multirow{2}{*}{$\begin{array}{c}\text { Параметры } \\
\text { кинетических } \\
\text { измерений }\end{array}$} & InP & InP $+\mathrm{QD}_{575}$ & InP-InAsP & InP-InAsP + QD \\
\cline { 2 - 5 } & & & & 0.7 \\
\hline$A_{1}$ & 0.58 & 0.36 & 1 & 4.8 \\
$\tau_{1}, \mathrm{~ns}$ & 0.6 & 0.5 & 2.8 & 0.3 \\
$A_{2}$ & 0.42 & 0.64 & - & 33.7 \\
$\tau_{2}, \mathrm{~ns}$ & 2.5 & 2.6 & - & 13.5 \\
$\langle\tau\rangle, \mathrm{ns}$ & 2.0 & 2.4 &
\end{tabular}

симум излучения QD). Эпитаксиальный рост НHК подробно описан в работах $[8,9]$. Как было установлено в работе [26], в спектрах люминесценции такой системы наблюдаются полосы в области $1.0-1.5 \mu \mathrm{m}$, кинетика которых зависит от состава лигандной оболочки. Полоса люминесценции в области $1.25-1.5 \mu \mathrm{m}$ принадлежит излучению QI InAsP, а полоса $1.0-1.2 \mu \mathrm{m}$ связана с радиальной квантовой ямой $(\mathrm{QW})$, которая образуется во время роста QI InAsP. B работе [25] приведены ПЭМ-изображения таких композиционных наноструктур.

При измерениях кинетики ФЛ на длине волны возбуждения $532 \mathrm{~nm}$ использовался импульсный $\mathrm{Nd}$ :YAG-лазер (длительность импульса $\sim 10 \mathrm{ps,} \mathrm{ча-}$ стота повторения импульсов $1 \mathrm{MHz}$ ), на длине волны возбуждения $635 \mathrm{~nm}$ - импульсный источник PicoQuant LDH-P-C-635B (длительность импульса $\sim 90 \mathrm{ps}$, частота следования $0.5-1 \mathrm{MHz}$ ). Регистрация сигнала ФЛ осуществлялась с помощью лавинного InGaAs/InP-фотодиода MicroPhotonDevices. Временное разрешение системы регистрации не превышало 100 ps. Кинетика ФЛ регистрировалась в максимумах полос люминесценции, соответствующих излучению ННК InP, а также QI и QW InAsP при температурах 77 и $300 \mathrm{~K}$.

\section{Результаты и обсуждение}

Результаты кинетических измерений приведены в табл. 1 и 2.

Таблица 1 иллюстрирует влияние коллоида QD на ФЛ InP. Как следует из данных, приведенных в [27] и собственных измерений, ФЛ InP сильно потушена за счет захвата возбужденных носителей заряда на низкоэнергетические ловушки. Нанесение коллоида QD на НHK InP мало влияет на кинетику ФЛ InP: обе компоненты затухания 0.5 и $2.5 \mathrm{~ns}$ практически не претерпевают изменений. При наличии включений InAsP (QI и QW) InP излучает из одного состояния со временем $3 \mathrm{~ns}$. Кинетика люминесценции InP существенно меняется при нанесении коллоида QD на НHК InP, содержащие QI и QW InAsP. Кинетика ФЛ ННК InP становится биэкспоненциальной со временами, близкими к временам излучения QD CdSe/ZnS [23]. Этот факт указывает, с одной стороны, на значительный вклад излучательного
Таблица 2. Возбуждение на длине волны $532 \mathrm{~nm}$. Регистрация на длине волны $1180(\mathrm{QW})$ и $1350 \mathrm{~nm}(\mathrm{QI})$

\begin{tabular}{c|c|c|c|c}
\hline Параметры & \multicolumn{2}{|c|}{ QI } & \multicolumn{2}{c}{ QI+ QD $545-\mathrm{TOPO}$} \\
\cline { 2 - 5 } $\begin{array}{c}\text { кинетических } \\
\text { измерений }\end{array}$ & $80 \mathrm{~K}$ & $300 \mathrm{~K}$ & $80 \mathrm{~K}$ & $300 \mathrm{~K}$ \\
\hline$A_{1}$ & 1 & 1 & 1 & 0.75 \\
$\tau_{1}, \mathrm{~ns}$ & 12.6 & 8.7 & 16 & 6.9 \\
$A_{2}$ & - & - & - & 0.25 \\
$A_{2}, \mathrm{~ns}$ & - & - & - & 54.8 \\
$\langle\tau\rangle, \mathrm{ns}$ & - & - & - & 18.9 \\
\hline$\lambda_{\mathrm{ex}}=532 \mathrm{~nm}$ & \multicolumn{3}{|c|}{$\mathrm{QW}$} & \multicolumn{2}{c}{$\mathrm{QW}+\mathrm{QD}_{545}$-TOPO } \\
\cline { 2 - 5 } & $80 \mathrm{~K}$ & $300 \mathrm{~K}$ & $80 \mathrm{~K}$ & $300 \mathrm{~K}$ \\
\hline$A_{1}$ & 0.5 & 1 & 0.67 & - \\
$\tau_{1}, \mathrm{~ns}$ & 2.8 & 2.2 & 6.6 & - \\
$A_{2}$ & 0.5 & - & 0.33 & - \\
$\tau_{2}, \mathrm{~ns}$ & 22.6 & - & 39.8 & - \\
$\langle\tau\rangle, \mathrm{ns}$ & 12.7 & - & 17.6 & Extinguished
\end{tabular}

или безызлучательного механизмов трансляции возбуждения с QD CdSe/ZnS на QW InAsP, сравнимого с прямым возбуждением, с другой стороны, на различие в каналах фононной дезактивации высших экситонных состояний InP и QW и QI InAsP [13,15], приводящий к резонансному механизму переноса энергии возбуждения с высших экситонных состояний QW и QI InAsP и QD $\mathrm{CdSe} / \mathrm{ZnS}$ на InP.

В табл. 2 представлены результаты кинетических измерений ФЛ QW и QI, из которых следует, что при возбуждении на длине волны $\lambda_{\mathrm{ex}}=532 \mathrm{~nm}$ люминесценция QI всегда затухает экспоненциально. Моноэкспоненциальность кинетики QI InAsP при возбуждении $532 \mathrm{~nm}$ и ее независимость от температуры подтверждает высказанное выше предположение о доминировании прямого заселения возбужденного состояния QI InAsP и отсутствие побочных ретрансляторов в фотодинамике возбуждения ННК на этой длине волны. Однако более важным является изменение кинетики ФЛ QI с одноэкспоненциальной на биэкспоненциальную и полное тушение люминесценции QW. Одной из причин первого является существование контактного тушения на гра- 
нице между QI InAsP и объемом InP, что показано в работе [9], где предложена модель кинетики процесса, учитывающая роль обратного переноса возбуждения с ловушек на излучающее состояние. Другим фактором является перенос энергии с QW на QI, обусловленный повышением температуры.

Возбуждение на длине волны $\lambda_{\mathrm{ex}}=633 \mathrm{~nm}$ делает (табл. 3) всю кинетику биэкспонециальной. Из данных таблицы следует, что кинетика ФЛ собственно QW и QI оказывается практически одинаковой при низких температурах, и при этом кинетика QI сохраняет свои параметры при ее увеличении, в то время как ФЛ QW с ростом температуры сильно тушится. Наличие коллоидной оболочки приводит как к росту общей длительности свечения QW и QI, так и особенному росту длительности и вклада второй компоненты свечения у QI. Такая подпитка излучающего состояния QI, возникающая при наличии коллоидной оболочки, может быть связана не только с обратным переносом возбуждения с близкорасположенных к QI ловушек, но и с новыми гибридными состояниями, образованными при взаимодействии лиганда с поверхностью наночастицы $[23,26,28,29]$. Такие состояния энергетически близки к излучающему состоянию и способны захватывать электроны или дырки. Последующая делокализация носителей (обратный перенос) на излучающие состояния может вести к увеличению длительности излучательной рекомбинации [10] и, следовательно, к росту интенсивности люминесценции.

В работе [29] обращено внимание на то, что увеличение длительности свечения и выхода люминесценции достигается при возбуждении в области прозрачности пассивирующего коллоида TOPO-CdSe/ZnS QD (635 и $1064 \mathrm{~nm}$ ). С этим эффектом связывается существенная роль эффекта обратного переноса. Лежащее в основе этого механизма предположение об образовании в результате пассивации гибридизированных состояний в запрещенной зоне нашло конкретное проявление в виде полосы свечения QI с $\lambda_{\max }$ в области $700 \mathrm{~nm}$. Такая полоса была обнаружена в работе [29] при возбуждении QI на длине волны $635 \mathrm{~nm}$ в присутствии оболочки из коллоида TOPO-CdSe/ZnS QD. Похожий эффект был обнаружен в [29] при возбуждении InP НHК на длине волны $1064 \mathrm{~nm}$. Это область поглощения как $\mathrm{QW}$, так и QI. Схема является прозрачной для коллоида $\mathrm{CdSe} / \mathrm{ZnS}-\mathrm{TOPO}$ и практически прозрачной для InP. Как видно из результатов, приведенных в [29], наличие оболочки из коллоида приводит к увеличению интенсивности свечения в несколько раз. При этом соотношение интенсивностей ФЛ образцов примерно одинаковое при накачке на длинах волн 532 и $1064 \mathrm{~nm}$. Длительность люминесценции InP ННК оставляет $5 \mathrm{~ns}$, что говорит о ее достаточно сильном тушении. Можно предположить, что причиной тушения является захват возбуждения на ловушки. Причем для QW это ловушки на межфазовой границе с InP. Для QI, как было показано в работе [5], основным механизмом тушения является так называемое

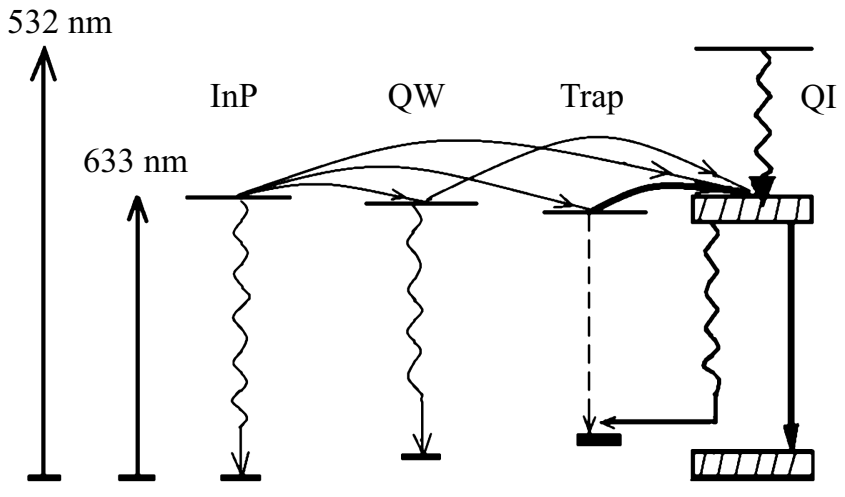

Диаграмма уровней, иллюстрирующая переходы между возбужденными состояниями (сплошные стрелки), а также пути релаксации возбуждения (волнистые стрелки) для фотогенерированных носителей в структуре коллоида ННК InP-TOPO-CdSe/ZnS при возбуждении длинами волн 532 и $633 \mathrm{~nm}$. Штриховая линия - люминесценция из низкоэнергетических ловушек.

Таблица 3. Возбуждение на длине волны $633 \mathrm{~nm}$. Регистрация на длине волны $1180(\mathrm{QW})$ и $1350 \mathrm{~nm}(\mathrm{QI})$

\begin{tabular}{c|c|c|c|c}
\hline Параметры & \multicolumn{2}{|c|}{ QI } & \multicolumn{2}{c}{ QI+ $\mathrm{QD}_{545}$-TOPO } \\
\cline { 2 - 5 } $\begin{array}{c}\text { кинетических } \\
\text { измерений }\end{array}$ & \multicolumn{2}{|c}{$300 \mathrm{~K}$} & $80 \mathrm{~K}$ & $300 \mathrm{~K}$ \\
\hline$A_{1}$ & 0.7 & 0.65 & 0.57 & \\
$\tau_{1}, \mathrm{~ns}$ & 6 & 28 & 14 & \\
$A_{2}$ & 0.3 & 0.35 & 0.43 & \\
$\tau_{2}, \mathrm{~ns}$ & 25 & 125 & 78 & \\
$\langle\tau\rangle, \mathrm{ns}$ & 12 & 62 & 42 & \\
\hline$\lambda_{\mathrm{ex}}=633 \mathrm{~nm}$ & \multicolumn{3}{|c}{$\mathrm{QW}$} & $\mathrm{QW}+\mathrm{QD}_{545}$-TOPO \\
\cline { 2 - 5 } & $80 \mathrm{~K}$ & $300 \mathrm{~K}$ & $80 \mathrm{~K}$ & $300 \mathrm{~K}$ \\
\hline$A_{1}$ & 0.76 & 0.60 & 0.57 & 0.60 \\
$\tau_{1}, \mathrm{~ns}$ & 4 & 1 & 9 & 4 \\
$A_{2}$ & 0.24 & 0.40 & 0.43 & 0.40 \\
$\tau_{2}, \mathrm{~ns}$ & 30 & 5 & 47 & 27 \\
$\langle\tau\rangle, \mathrm{ns}$ & 10 & 3 & 25 & 13
\end{tabular}

контактное тушение, связанное с образованием ловушек вблизи QI HНК в результате деформаций, вызванных фазовым переходом второго рода. Разумно предположить, что увеличение интенсивности свечения связано с увеличением вероятности переноса возбуждения с QW на QI, вызванного текстурными перестройками при образовании лигандной оболочки. Этот канал ретрансляции возбуждения может значительно усилиться за счет обратного переноса. Иллюстрацией к вышесказанному служит рисунок, где схематически показана обобщенная картина фотодинамики возбуждения в исследованной системе с точки зрения представлений модели обратного переноса. Таким образом, фотодинамика обратного переноса состоит из двух стадий: быстрого накопления возбуждения на ловушках разного типа и растянутого во времени высвобождения носителей и их излучательной рекомбинации. 


\section{Заключение}

В настоящей работе, основываясь на спектрокинетическом подходе, рассмотрено влияние оболочки коллоидных QD CdSe/ZnS на фотодинамику гибридной полупроводниковой наноструктуры InP/InAsP/InP при возбуждении разными длинами волн. Моноэкспоненциальность кинетики QI InAsP при возбуждении на длине волны 532 $\mathrm{nm}$ и ее независимость от температуры подтверждают предположение о доминировании прямого заселения возбужденного состояния QI InAsP и отсутствии побочных ретрансляторов в фотодинамике возбуждения ННК на этой длине волны.

Подтверждено существование в исследуемой наноструктуре двух типов ловушечных состояний: на границе внутреннего гетероперехода и образованных за счет поверхностно-лигандных взаимодействий. Предположено, что локализация электронов на антисвязывающих орбиталях в системе $\mathrm{CdSe} / \mathrm{ZnS}$-TOPO играет заметную роль в увеличении времени жизни ФЛ QI. С учетом работ $[10,25,29]$ полученные результаты подтверждают заметное влияние эффекта обратного переноса на интенсивность свечения, компенсирующее тушение возбужденных состояний, связанное с захватом возбуждения на низкоэнергетические ловушки. В этом же контексте обсуждены результаты, полученные в [29] при возбуждении наноструктуры на длинах волн 633 и $1064 \mathrm{~nm}$ в области прозрачности коллоида.

\section{Благодарности}

Авторы выражают свою признательность А.И. Хребтову, Е.Н. Бодунову и А.С. Кулагиной за полезное обсуждение работы.

\section{Конфликт интересов}

Авторы заявляют об отсутствии конфликта интересов.

\section{Список литературы}

[1] Zieliński M. // Phys. Rev. 2013. V. 88. P. 115424. doi.org/10.1103/PhysRevB.88.115424

[2] Leandro Lorenzo, Gunnarsson Christine P., Reznik R., Jöns K.D., Shtrom I., Khrebtov A., Kasama Takeshi, Zwiller V., Cirlin G., Akopian N. // Nano Lett. 2018. V. 18. N 11. P. 7217-7221. doi 10.1021/acs.nanolett.8b03363

[3] Chen Yan, Zadeh I.E., Jöns K.D., Fognini A., Reimer M.E., Zhang J., Dalacu Dan, Poole P.J., Ding F., Zwiller V., Schmidt O.G. // Appl. Phys. Lett. 2016. V. 108. P. 182103. doi 10.1063/1.4948762.

[4] Dalacu Dan, Poole Philip J., Williams Robin L. // Nanotechnology. 2019. V. 30. N 23. P. 232001. doi 10.1088/1361-6528/ab0393

[5] Kulagina A.S., Khrebtov A.I., Reznik R.R., Ubyivovk E.V., Litvin A.P., Skurlov I.D., Cirlin E., Bodunov E.N., Danilov V.V. // Opt. Spectrosc. 2020. V. 128. P. 119-124. doi 10.1134/S0030400X20010129
[6] Jones M., Scholes D.G. // J. Mater. Chem. 2010. V. 20. P. 3533-3538.

[7] Yeltik Aydan, Olutas Murat, Sharma Manoj, Gungor Kivanc, Demir Hilmi Volkan. // J. Phys. Chem. C. 2019. V. 123. P. 1470-1476.

[8] Cirlin G.E., Shtrom I.V., Reznik R.R., Samsonenko Y.B., Khrebtov A.I., Bouravleuv A.D., Soshnikov I.P. // Semiconductors. 2016. V. 50. P. 1421-1424. doi 10.1134/S1063782616110257.

[9] Khrebtov A.I., Reznik R.R., Ubyivovk E.V., Litvin A.P., Skurlov I.D., Parfenov P.S., Kulagina A.S., Danilov V.V., Cirlin G.E. // Semiconductors. 2019. V. 53. N 9. P. $1258-1261$. doi 10.1134/S1063782619090082

[10] Fischer Sean A., Crotty A.M., Kilina S.V., Ivanov S.A., Tretiak S. // Nanoscale. 2012. V. 4. P. 904. doi $10.1039 / \mathrm{c} 2 \mathrm{nr} 11398 \mathrm{~h}$

[11] Williams E.S., Major K.J., Tobias A., Woodall D., Morales V., Lippincott C., Moyer P.J., Jones M. // J. Phys. Chem. C. 2013. V. 117. P. 4227-4237.

[12] Woodall D.L., Tobias A.K., Jones M. // Chem. Phys. 2016. V. 471. N 2. doi 10.1016/j.chemphys.2015.10.011

[13] Nikiforov V.G. // Chem. Phys. 2020. V. 538. P. 110916. doi 10.1016/j.chemphys.2020.110916

[14] Knowles K.E., Tice D.B., McArthur E.A., Solomon G.C., Weis E.A. // J. Am. Chem. Soc. 2010. V. 132. P. 1041-1050. doi $10.1021 /$ ja907253s

[15] Palato S., Seiler H., McGovern L., Mack T.G., Jethi Lakshay, Kambhampati Patanjali. // J. Phys. Chem. C. 2017. V. 121. P. 26519. doi 10.1021/acs.jpcc.7b09145

[16] Danilov V.V., Kulagina A.S., Sibirev N.V. // Appl. Optics. 2018. V. 7. N 28. P. 8166-8170. doi 10.1364/AO.57.008166

[17] Orlova A.O., Kurochkina M.A., Gromova Yu.A., Maslov V.G., Bodunov E.N., Baranov A.V., Fedorov A.V. // Proc. SPIE. 2014. V. 9126. P. 912617(10). doi 10.1117/12.2052170

[18] Hernandez-Martinez P.L., Govorov A.O., Demiz H.V. // J. Phys. Chem. C. 2014. V. 118. P. 4951. doi 10.1021/jp409833b

[19] Bodunov E.N., Danilov V.V., Panfutova A.S., Simões Gamboa A.L. // Ann. Phys. (Berlin). 2016. V. 528. N 3-4. P. 272. doi 10.1002/andp. 201500350

[20] Bodunov E.N., Simões Gamboa A.L. // Semiconductors. 2018. V. 52. N 5. P. 587. doi 10.1134/S1063782618050044

[21] Bodunov E.N., Simões Gamboa A.L. // Semiconductors. 2019. V. 53. N 16. P. 2133. doi 10.1134/S1063782619120078

[22] Stroyuk O., Raevskaya A., Spranger F., Gaponik N., Zahn D.R.T. // ChemPhysChem. 2019. V. 20. N 12. P. 1640-1648. doi 10.1002/cphc.201900088

[23] Danilov V.V., Panfutova A.S., Khrebtov A.I., Titova T.S. // Opt. Spectrosc. 2015. V. 118. N 1. P. 94-98. doi 10.1134/S0030400X15010099

[24] Berberan-Santos M.N., Bodunov E.N., Valeur B. // Chem. Phys. 2005. V. 315. P. 171-182. doi 10.1016/j.chemphys.2005.04.006

[25] Khrebtov A.I., Kulagina A.S., Danilov V.V., Gromova E.S., Skurlov I.D., Litvin A.P., Reznik R.R., Shtrom I.V., Cirlin G.E. // Semiconductors. 2020. V. 54. P. 1141-1146.

[26] Rabouw F.T., vander Bok J.C., Spinicelli Piernicola, Mahler Benoît, Nasilowski M., Pedetti Silvia, Dubertret Benồ t, Vanmaekelbergh D. // Nano Lett. 2016. V. 16. N 3. P. 2047-2053. doi 10.1021/acs.nanolett.6b0005323. 
[27] van Vugt Lambert K., Veen Sandra J., Bakkers E.P.A.M., Roest A.L., Vanmaekelbergh D. // J. Am. Chem. Sos. 2005. V. 127. P. $12357-12362$.

[28] Califano M., Franceschetti A., Zunger A. // Nano Lett. 2005. V. 5. N 12. P. 2360-2364. doi 10.1021/n1051027p

[29] Khrebtov A.I., Danilov V.V., Kulagina A.S., Reznik R.R., Skurlov I.D., Litvin A.P., Safin F.M., Gridchin V.O., Shevchuk D.S., Shmakov S.V. et al. // Nanomaterials. 2021. V. 11. P. 640. doi 10.3390/nano11030640 